\title{
Prescription of
}

\section{antidepressants and the risk of road traffic crash in the} elderly: a case-crossover study

Ludivine Orriols, ${ }^{1,2}$ Machelle Wilchesky, ${ }^{3,4,5}$ Emmanuel Lagarde ${ }^{1,2} \&$ Samy Suissa ${ }^{4}$

${ }^{1}$ University Bordeaux, ISPED, ${ }^{2}$ INSERM, Equipe PPCT, ISPED, Centre INSERM

U897-Epidemiologie-Biostatistique, Bordeaux, France, ${ }^{3}$ Donald Berman Maimonides Geriatric Center, McGill University, ${ }^{4}$ Center for Clinical Epidemiology, Lady Davis Institute, Jewish General Hospital, Department of Epidemiology and Biostatistics and ${ }^{5}$ Department of Medicine, McGill University, Montreal, Quebec, Canada

\section{Correspondence}

Dr Ludivine Orriols, Equipe Prévention et prise en charge des traumatismes, Centre de recherche INSERM U897

'Epidémiologie et Biostatistiques'

Université Bordeaux Segalen, Case 11, 146 rue Léo Saignat, 33076 Bordeaux Cedex, France.

Tel.: +335 57571504

Fax:+335 57571504

E-mail:

ludivine.orriols@isped.u-bordeaux2.fr

Keywords

antidepressants, case-crossover, elderly, epidemiology, traffic crashes

Received

22 June 2012

Accepted

3 February 2013

\section{WHAT IS ALREADY KNOWN ABOUT THIS SUBJECT}

- Antidepressants are often used by persons over age 65 years and may lead to an increased risk of crash.

- Results of epidemiological studies have been inconclusive owing to different study designs.

\section{WHAT THIS STUDY ADDS}

- The risk of crash is increased in drivers with a prescription of antidepressants before their crash when compared with prescription of antidepressants 4 to 8 months before the crash $(\mathrm{OR}=1.19,95 \% \mathrm{Cl}$ $1.08,1.30$ to $1.42,95 \% \mathrm{Cl} 1.30,1.55)$.

- This risk is likely to be linked to depression symptoms.

\section{AIM}

To investigate the impact of antidepressants on the risk of road traffic crash in the elderly.

\section{METHODS}

Reports from the Universal Quebec Automobile Insurance Agency database were matched with data on antidepressant prescription from the Quebec Health Insurance Agency. The case-crossover analysis consisted in comparing exposure during a period immediately before the crash with exposure during earlier periods, for the same subject.

\section{RESULTS}

One hundred and nine thousand four hundred and six drivers between 66 and 84 years of age involved in a traffic crash between 1988 and 2000 were included. Two thousand nine hundred and nineteen (2.7\%) were exposed to an antidepressant on the day of the crash.

Case-crossover analysis found an increased risk of crash in drivers with a prescription of antidepressants before their crash when compared with a prescription of antidepressants 4 to 8 months before the crash $(\mathrm{OR}=1.19,95 \% \mathrm{Cl} 1.08,1.30$ to $1.42 .95 \% \mathrm{Cl} 1.30,1.55)$. With the most recent control periods, results were not significant.

\section{CONCLUSION}

A patient's mental state is probably more similar between two periods that are close to each other than up to 8 months before. Consequently, the risk of crash is likely to be linked to symptoms of depression. 


\section{Introduction}

Antidepressants are commonly used worldwide. In the province of Quebec, Canada, the prevalence of antidepressant use was $10.6 \%$ in 2004 and was even higher in the elderly (12.8\% in the 65-74 year old age group and $15.3 \%$ in the 75 year old and more group) [1]. Experimental studies have suggested that antidepressants may impair driving abilities [2-9]. While a few epidemiological studies on the risk of road traffic crashes associated with the use of antidepressants have been conducted, they are difficult to compare owing to the different study designs. One cohort study [10] and another case-control study [11], both conducted in older drivers found a significant association between the risk of being involved in a car crash and exposure to tricyclic antidepressants ( $R R=2.2$, 95\% Cl 1.3, 3.5 and OR $=2.3,95 \% \mathrm{Cl} 1.1,4.8$, respectively). Bramness et al. found an increased risk for drivers who had received a prescription for sedative antidepres sants $(\mathrm{SIR}=1.4,95 \% \mathrm{Cl} 1.2,1.6)$ or non-sedative antidepressants (SIR $=1.6,95 \% \mathrm{Cl} 1.5-1.7)$ [12]. In another study of 19386 drivers aged 18 years and older, Barbone et al. found no relationship with the risk of traffic crash associated with either selective serotonin receptor inhibitors or with tricyclic antidepressants [13]. A pharmacoepidemiological study of the effect of benzodiazepines on the risk of road traffic crash compared results of a case-control study and of a case-crossover study using the same prescription claims database. The case-control analysis showed an increased risk of crash with current use of long half-life benzodiazepines. The stratified casecrossover analysis demonstrated an effect of infrequent use on the risk of crash and no significant impact in frequent users. The stratified case-control analysis showed similar results in both subgroups [14]. A recent casecrossover study showed that the risk of crash was increased in users who initiated an antidepressant treatment and a case-control analysis showed an increased risk of responsibility in the crash for all antidepressant users [15]. Risk estimates in case-control studies are a combination of the effect of medicines in regular users, the effect of medicines in occasional users and the effect of depression symptoms. Indeed, confounding by indication remains an issue in such studies. The only way to avoid this bias would be to adjust for the presence and gravity of depression symptoms. However, this information is often lacking in administrative databases. The case-crossover design is an answer to this issue as it controls for individual characteristics given that the subject acts as his own control.

The aim of our present study was to apply the case-crossover method to investigate the impact of antidepressants on the risk of road traffic crash in the elderly, using prescription claims database and records of crashes in the province of Quebec, Canada.

\section{Methods}

Participants

All drivers between 66 and 84 years of age involved in a first road traffic crash during the cohort period between 1988 and 2000 were included. An upper age of 84 years was chosen because individuals are less likely to be driving beyond this age [16].

The universal drug prescription programme in the province of Quebec covers residents of the province aged 65 years old and more. In order to have a complete 1 year history of medicine exposure, participants must have been 66 years of age and older to be included.

\section{Data sources}

Drivers involved in a road traffic crash were identified using the Universal Quebec Automobile Insurance Agency database which records all reports of crashes in the province and contains information on crashes, including the date of the crash.

Antidepressant use was obtained from the Quebec Health Insurance Agency, the agency responsible for administering insured health care services for the province. The prescription drug files contain data pertaining to all filled outpatient prescriptions which were prescribed by a Québec-licensed physician and filled at community pharmacies for medications listed on the liste de medicaments. The province's formulary includes the date of dispensing, the International Nonproprietary Name and the duration of treatment prescribed. The two databases are linked using a unique ID number.

\section{Outcome}

The outcome was defined as all crashes occurring between 1988 and 2000, including injurious crashes and crashes with property damage only.

\section{Exposure to antidepressants}

Exposure was considered to start on the day following dispensing because only part of the day of dispensing would be exposed time. This also excludes antidepressants that may be prescribed as a consequence of the crash. The duration of treatment, as registered in the Quebec Health Insurance Agency database was applied to obtain the end date of treatment.

Antidepressants were studied as an all-inclusive drug category, and then also according to the three following classes: tricyclic antidepressants, selective serotonin re-uptake inhibitors (SSRIs) and all other antidepressants.

\section{Study design}

The case-crossover analysis consists of a pair-matched analytical approach to compare exposure during a period immediately before the crash (case period) with exposure during earlier periods (controls periods) for the same 
subject [17]. As in matched case-control studies, only subjects with discordant exposures in the case and control periods participate in analysis. This design is thus appropriate to study the effect of intermittent exposures while controlling for time-invariant characteristics given that the subject acts as his own control.

We compared antidepressant exposure on the day of the crash with antidepressant exposure on eight 1 day control periods, each respectively separated from the case period by a 30 day, 60 day, 90 day, 120 day, 150 day, 180 day, 210 day and 240 day wash-out period.

A sub-analysis was performed in order to study the impact of new use of an antidepressant treatment. A subgroup of new users was thus defined as drivers exposed any day in the first 30 days exposure to an antidepressant, following a 6 month period with no exposure. In order to allow drivers to be newly exposed in the control period, as well as in the case period, the control period had to be separated from the case period by a 240 day wash-out period. For statistical power reasons, antidepressants were studied as an all inclusive drug category in this sub-analysis.

Odds ratios were estimated by conditional logistic regression, using the PHREG procedure in the SAS statistical software package, version 9.2 (SAS Institute Inc, Cary, NC, USA).

\section{Ethical approval}

The study protocol was approved by the Ethics Committee of the Jewish General Hospital. All data used in this study were anonymized.

\section{Results}

\section{Study population}

One hundred and nine thousand four hundred and six drivers aged between 66 and 84 years were involved in a first road traffic crash during the cohort period, between 1988 and 2000. The mean age on the day of the crash was 71.9 years $(S D=4.6)$ and $80.5 \%$ were men. Among them, 21943 (20.1\%) had at least one prescription of an antidepressant over the period considered and 2919 (2.7\%) were exposed to an antidepressant on the day of the crash. The distribution of exposures according to the class of antidepressants is presented in Table 1.

\section{Case-crossover analysis}

The results showed that the longer the wash-out period was, the greater the risk of crash was increased in users of all antidepressants. The number of discordant pairs participating in the analysis increased too. The same pattern was observed in SSRIs users (Table 2).

The case-crossover analysis showed an increased risk of crash in new users $(\mathrm{OR}=1.30,95 \% \mathrm{Cl} 1.08,1.57)$ that was

\section{Table 1}

Exposure to antidepressants on the day of the crash

\begin{tabular}{|lrc|}
\hline Antidepressants & $\boldsymbol{n}$ & $\%$ \\
\hline All & 2919 & 100 \\
\hline Tricyclics & 1815 & 62.2 \\
Amitriptyline & 746 & 25.6 \\
Amoxapine & 8 & 0.3 \\
Clomipramine & 69 & 2.4 \\
Desipramine & 73 & 2.5 \\
Doxepine & 328 & 11.2 \\
Imipramine & 122 & 4.2 \\
Maprotiline & 84 & 2.9 \\
Nortriptyline & 71 & 2.4 \\
Protriptyline & 2 & 0.1 \\
Trimipramine & 329 & 11.3 \\
SSRls* & 882 & 30.2 \\
Citalopram & 19 & 0.7 \\
Fluoxetine & 214 & 7.3 \\
Fluvoxamine & 107 & 3.7 \\
Paroxetine & 270 & 9.2 \\
Sertraline & 275 & 9.4 \\
Others & 286 & 9.8 \\
Bupropion & 3 & 0.1 \\
Moclobemide & 19 & 0.7 \\
Nefazodone & 25 & 0.9 \\
Phenelzine & 12 & 0.4 \\
Tranylcypromine & 7 & 0.2 \\
Trazodone & 165 & 5.7 \\
Venlafaxine & 62 & 2.1 \\
\hline
\end{tabular}

*Escitalopram was approved by the US Food and Drug Administration in 2002, after the 1988-2000 cohort period.

not different from the risk estimated in other drivers who were not new users (OR $=1.49,95 \% \mathrm{Cl} 1.35,1.65)$.

\section{Discussion}

This study found evidence for an increased risk of road traffic crash in older drivers who had a prescription of antidepressants before their crash when compared with a prescription of antidepressants 4 to 8 months before the crash (OR $=1.19,95 \% \mathrm{Cl} 1.08,1.30$ to $1.42 .95 \% \mathrm{Cl} 1.30,1.55)$. Case-crossover analysis showed that the risk is likely to be linked to depression symptoms.

The case-crossover design requires that the exposure be both intermittent and have a transient effect. These assumptions may not actually be met in practice for antidepressant use among older drivers. Chronic users who were exposed all along the period of time considered were excluded from the case-crossover analysis. This design is thus likely to underestimate the risk in people using chronic medications. An underlying assumption when comparing the case and the control periods is that each subject maintains the same driving pattern in both the exposed and unexposed periods. This is why drivers over 84 years old were excluded as they are less likely to be driving beyond this age [16] so the probability of 


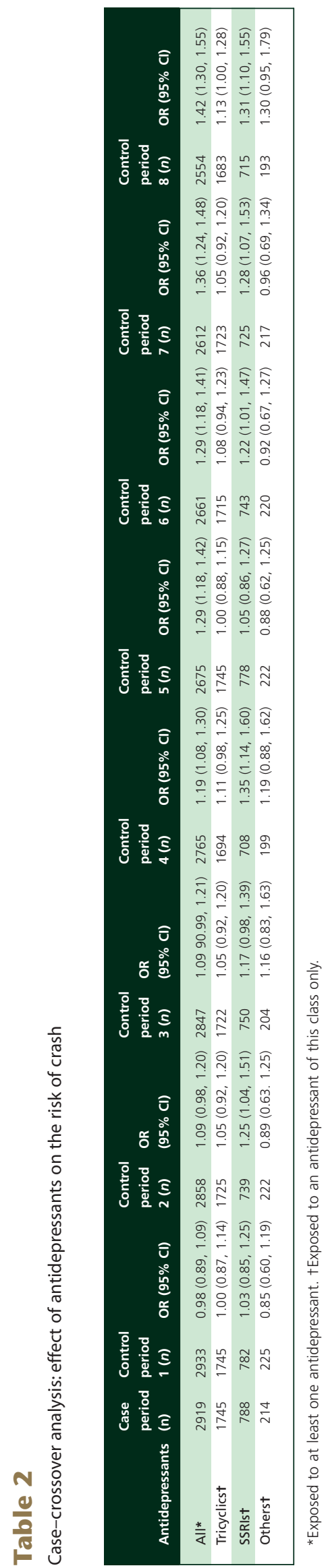

maintaining the same driving pattern in both the case and the control periods is low. To the extent that drivers did not drive during control periods, the study may underestimate the risk of crash. While controlling for time-invariant characteristics such as chronic diseases, the case-crossover design does not control for unmeasured variation within an individual. Results from sensitivity analyses suggest that the increased risk of crash is likely to be linked to the presence of depression. Indeed, the analyses which included the most recent control periods were not significant. It seems that the patient's mental state was more similar between two periods that are close to each other, than up to 240 days previously. The case-crossover design can be implemented as a new user design. Ray discussed the usefulness of such designs in observational studies [18]. Conducting registry-based studies reduces the logistical barriers to new user designs as detailed drug prescription is provided in health care databases. However, in order to conduct such analysis, the control period has to be far from the case period so the effect of depression symptoms cannot be excluded. Indeed, the longer the study period considered, the greater the potential for confounding by time-varying factors.

Drug exposure was ascertained from computerized records. These data were not subject to under-reporting, a major problem encountered when medicine exposure data are self-reported [19]. However, while we know that a prescription has been filled, we do not have any information pertaining to prescription adherence and we assumed that subjects were exposed to antidepressants for the prescribed duration of their treatment. Non-adherence, which we cannot verify, would result in exposure misclassification. However, the resulting misclassification would most likely be non-differential.

Other studies were interested in antidepressants and the risk of crash in the elderly $[10,11]$. These two epidemiological studies only looked at the impact of tricyclic antidepressants on the risk of crash as SSRIs were developed later. In a case-control study, current users of tricyclic antidepressants (with a prescription filled within 60 days before the index date) were at increased risk of injurious road traffic crash ( $\mathrm{OR}=2.3,95 \% \mathrm{Cl} 1.1,4.8)$ while no significant association was found in users exposed between 60 days and 6 months before the crash [11]. Bramness et al. found similar risk estimates among incident users and prevalent users [12]. Unlike the Leveille et al.'s study [11], the results were not adjusted for the diagnosis of depression. The case-crossover study conducted by Barbone et al. should be more comparable with our study as the same design was used [13]. This study found no association between antidepressants (tricyclics or SSRIs) and the risk of crash. However, the absence of an association may be explained by a lack of statistical power since the number of older drivers exposed to antidepressants was low (38 older drivers exposed to tricyclics and five exposed to SSRIs). Ravera et al. also conducted a case-crossover study on the 
impact of SSRIs and other antidepressants. The analysis among acute users did not show any association with the risk of crash. However, the authors noted that their study might not have had adequate statistical power [20]. The most recent epidemiological study, conducted in France, used a case-crossover design and showed that the risk of crash was increased after an initiation of antidepressant treatment $(\mathrm{OR}=1.49,95 \% \mathrm{Cl} 1.24,1.79)$ [15]. This latter study included drivers of all ages. It is likely that, in our study, older drivers refrain themselves from driving when taking antidepressants for the first time. Results by classes of antidepressants suggested that the depressive state of older drivers using tricyclics was more stable than in SSRI users.

Experimental studies conducted on healthy volunteers have shown a deleterious effect on driving of amitriptyline $[2,4]$. It is interesting to note that amitriptyline and trimipramine, which are tricyclics, were the antidepressants the most commonly used in our study. Nowadays, they are mainly given to treat anxiety and resistant depression. Paroxetine [5-8] and fluoxetine [3], both SSRIs, appear to have a smaller potential for impairing performance. The experimental studies were conducted in young, healthy volunteers who are very different from the population of drivers included in the present study who are older and depressed. Depression is associated with a slower reaction time in a driving simulator [21] and with lower scores in attention abilities [22]. Worrying thoughts in depression seem to be predominant on other important information [23]. It has been shown that treated depressed patients had better performances on tasks related to driving than untreated depressed patients [24]. Long term treatment with antidepressants may thus reduce the risk but not to baseline, the residual risk being linked to symptoms of depression.

Our study provides further insight into the impact of the prescription of antidepressants on traffic safety for the elderly. Patients and prescribers should be warned about this risk.

\section{Competing Interests}

All authors have completed the Unified Competing Interest form at http://www.icmje.org/coi_disclosure.pdf (available on request from the corresponding author) and declare: no support from any organization for the submitted work, no financial relationships with any organizations that might have an interest in the submitted work in the previous 3 years and no other relationships or activities that could appear to have influenced the submitted work.

The authors thank the staff of the Régie de l'assurance maladie du Québec (RAMQ) and of the Société de l'assurance automobile du Québec (SAAQ) for their expertize and assistance in obtaining the databases.
This research was funded by a grant from the Canadian Institutes of Health Research (CIHR). The funder had no role in the study.

\section{REFERENCES}

1 Conseil du médicament. Usage des antidépresseurs chez les personnes inscrites au régime public d'assurance médicaments du Québec. Etude descriptive 1999-2004. 2008.

2 Hindmarch I, Subhan Z, Stoker MJ. The effects of zimeldine and amitriptyline on car driving and psychomotor performance. Acta Psychiatr Scand Suppl 1983; 308: 141-6.

3 Kerr JS, Fairweather DB, Hindmarch I. Effects of fluoxetine on psychomotor performance, cognitive function and sleep in depressed patients. Int Clin Psychopharmacol 1993; 8: 341-3.

4 Kerr JS, Powell J, Hindmarch I. The effects of reboxetine and amitriptyline, with and without alcohol on cognitive function and psychomotor performance. Br J Clin Pharmacol 1996; 42: 239-41.

5 Raptopoulos P, McClelland GR, Jackson D. The clinical pharmacology of paroxetine in healthy subjects. Acta Psychiatr Scand Suppl 1989; 350: 46-8.

6 Ridout F, Meadows R, Johnsen S, Hindmarch I. A placebo controlled investigation into the effects of paroxetine and mirtazapine on measures related to car driving performance. Hum Psychopharmacol 2003; 18: 261-9.

7 Robbe HW, O'Hanlon JF. Acute and subchronic effects of paroxetine 20 and $40 \mathrm{mg}$ on actual driving, psychomotor performance and subjective assessments in healthy volunteers. Eur Neuropsychopharmacol 1995; 5: 35-42.

8 Warrington SJ, Dana-Haeri J, Sinclair AJ. Cardiovascular and psychomotor effects of repeated doses of paroxetine: a comparison with amitriptyline and placebo in healthy men. Acta Psychiatr Scand Suppl 1989; 350: 42-4.

9 Wingen M, Bothmer J, Langer S, Ramaekers JG. Actual driving performance and psychomotor function in healthy subjects after acute and subchronic treatment with escitalopram, mirtazapine, and placebo: a crossover trial. J Clin Psychiatry 2005; 66: 436-43.

10 Ray WA, Fought RL, Decker MD. Psychoactive drugs and the risk of injurious motor vehicle crashes in elderly drivers. Am J Epidemiol 1992; 136: 873-83.

11 Leveille SG, Buchner DM, Koepsell TD, McCloskey LW, Wolf $\mathrm{ME}$, Wagner EH. Psychoactive medications and injurious motor vehicle collisions involving older drivers. Epidemiology 1994; 5: 591-8.

12 Bramness JG, Skurtveit S, Neutel Cl, Morland J, Engeland A. Minor increase in risk of road traffic accidents after prescriptions of antidepressants: a study of population registry data in Norway. J Clin Psychiatry 2008; 69: e1-e5.

13 Barbone F, McMahon AD, Davey PG, Morris AD, Reid IC, McDevitt DG, MacDonald TM. Association of road-traffic accidents with benzodiazepine use. Lancet 1998; 352: 1331-6. 
14 Hebert C, Delaney JA, Hemmelgarn B, Levesque LE, Suissa S. Benzodiazepines and elderly drivers: a comparison of pharmacoepidemiological study designs. Pharmacoepidemiol Drug Saf 2007; 16: 845-9.

15 Orriols L, Queinec R, Philip P, Gadegbeku B, Delorme B, Moore N, Suissa S, Lagarde E. Risk of injurious road traffic crash after prescription of antidepressants. J Clin Psychiatry 2012; 73: 1088-94.

16 Dellinger AM, Sehgal M, Sleet DA, Barrett-Connor E. Driving cessation: what older former drivers tell us. J Am Geriatr Soc 2001; 49: 431-5.

17 Maclure M. The case-crossover design: a method for studying transient effects on the risk of acute events. Am J Epidemiol 1991; 133: 144-53.

18 Ray WA. Evaluating medication effects outside of clinical trials: new-user designs. Am J Epidemiol 2003; 158: 915-20.

19 Honkanen R, Ertama L, Linnoila M, Alha A, Lukkari I, Karlsson $\mathrm{M}$, Kiviluoto O, Puro M. Role of drugs in traffic accidents. $\mathrm{Br}$ Med J 1980; 281: 1309-12.
20 Ravera S, van Rein N, de Gier JJ, de Jong-van den Berg LT. A comparison of pharmacoepidemiological study designs in medication use and traffic safety research. Eur J Epidemiol 2012; 27: 473-81.

21 Bulmash EL, Moller HJ, Kayumov L, Shen J, Wang X, Shapiro CM. Psychomotor disturbance in depression: assessment using a driving simulator paradigm. J Affect Disord 2006; 93: 213-8.

22 Baune BT, Miller R, McAfoose J, Johnson M, Quirk F, Mitchell $D$. The role of cognitive impairment in general functioning in major depression. Psychiatry Res 2010; 176: 183-9.

23 Joormann J, Lira Yoon K, Zetsche U. Cognitive inhibition in depression. Appl Prev Psychol 2007; 12: 128-39.

24 Brunnauer A, Laux G, David I, Fric M, Hermisson I, Moller HJ. The impact of reboxetine and mirtazapine on driving simulator performance and psychomotor function in depressed patients. J Clin Psychiatry 2008; 69: 1880-6. 